\title{
Rehabilitation of the Stomatognathic System in Patients with Hemiglosectomy. A Case Report
}

\section{Montes González Jesús*, Peso Florencia, Valenzuela María Eva, Rimoldi Candela, Felice Agostina, Provassi Paula}

Kinesiology Unit, Pedro Fiorito General Acute Interzonal Hospital, Buenos Aires, Argentina

*Corresponding Author: Montes González Jesús, Kinesiology Unit, Pedro Fiorito General Acute Interzonal Hospital, Buenos Aires, Argentina.
Received: October 13, 2021

Published: January 31, 2022

(C) All rights are reserved by Montes González Jesús., et al.

\begin{abstract}
Introduction: Head and neck cancer includes tumors of the oral cavity, pharynx and larynx, with oral cancer being the eighth most common in the world. The tongue is one of the most common sites of carcinoma oral. The primary treatment of choice for this pathology is surgical by hemiglosectomy. This type of surgery affects the stomatognathic system which ensures the vital functions of swallowing, chewing and communication. As for the rehabilitation treatment, the literature proposes exercises to improve the musculature and mobility of the tongue and the floor of the mouth as well as the jaw. The objective of this study is to describe the evolution of a patient with hemiglosectomia without indication of prosthesis or reconstruction, after having carried out a rehabilitation plan of the stomatognathic system in the external office of higA Pedro Fiorito de Avellaneda.

Case Description: Male patient of 32 years with diagnosis of left hemiglosectomy secondary to oral carcinoma. At the initial evaluation, it presents alterations for masticatory and lingual movements and decreased cervical and scar mobility. As for their quality of life and stab altered. There are 2 sessions per week of 1 hour duration with various exercises of elongation, muscle energy, trigger points, active and passive exercises among others. Upon re-evaluation, the patient achieves improvement in terms of the mobility of the altered structures and their quality of life.

Conclusion: The present work describes the evolution of a patient with hemiglosectomy after performing kinetic treatment of the stomatognathic system in the external office of higA Pedro Fiorito de Avellaneda.
\end{abstract}

Keywords: Hemiglosectomy; Tongue Cancer; Stomatognathic System; Rehabilitation

\section{Introduction}

Head and neck cancer includes tumors of the oral cavity, pharynx, and larynx. The risk factors that are related are the consumption of alcohol and tobacco [1]. The occurrence of oral cancer is particularly high in men, the eighth most common worldwide, with lengua being one of the most frequent sites of oral carcinoma. Incidence rates range from 1 to 10 cases per 100,000 population in many countries [2]. The primary treatment of choice for this pathology is surgical. Hemiglosectomy is the resection of half of the floor of the mouth and tongue, and may involve the base of the same [3]. This resection not only reduces lingual volume but also affects the mobility of the remaining musculature, speech articulation and swallowing, particularlyin the oral phases [4].
According to the paper published by Mannelli in 2017, surgical classification for tongue defects focuses on functional subunits that require reconstruction. It is divided into five groups in ascending order of reconstructive complexity. Under this system, type 1 and 2 defects mainly require the restoration of the shape of the tongue, considering that more than $50 \%$ of the mobile body can be preserved with good functional expectations [5].

Regarding rehabilitation treatment, the collected literature proposes exercises, both to improve the musculature and mobility of the tongue and floor of the mouth as well as the jaw; among them, strength training, resistance, and coordination; and technical stimulation for patients with delayed firing of the swallowing reflex [6 8]. The stomatognathic system is a subsystem of the organ system 
which ensures the vital functions of nutrition: breathing, suction, swallowing, chewing and communication. Involucra according to Manns and Díaz to the structures ranging from the supraorbital ridge to the hyoid bone and according to Rocavado includes the shoulder girdle, since it considers the structures of head and neck as an indivisible functional unit [9]. The articles found do not specify treatments devoted to it as a whole. In addition, many of them focus only on pre-dental prosthesis rehabilitation, and pre-lingual reconstruction. Therefore, the objective of this study is to describe the evolution of a patient with hemiglosectomy without indication of prosthesis or reconstruction, after having carried out a rehabilitation plan of the stomatognathic system in the external office of higa Pedro Fiorito de Avellaneda.

\section{Case Description}

A 32-year-old male patient with a history of smoking and social enolism who was admitted on May 29, 2019 to the HIGA Pedro
Fiorito guard for a surgery scheduled for a clinical picture of two months of evolution debido to a squamous cell carcinoma on the floor of the mouth with lingual involvement. The next day, he underwent a left hemiglosectomy with supraomohioid cervical nodal emptying through a laterocervicalincision, with dissection of the superficial aponeurotic muscle and preservation of the posterior, hypoglossal and lingual atrial nerves. Due to the surgery, a surgical tracheostomy was performed to ensure ventilation during the surgical act. The patient returns to the gener roomtolucid, afebrile and hemodynamically stable and on June 3 begins the decanulation process in charge of the kinesiology team, achieving decanular 4 days later. After discharge, he is referred to the external kinesiology office where the initial evaluation was evaluated and rehabilitation began. Figure 1 describes the important events in the patient's evolution from diagnosis to final evaluation. The patient signed the informed consent for the conductof this study (Annex 1).

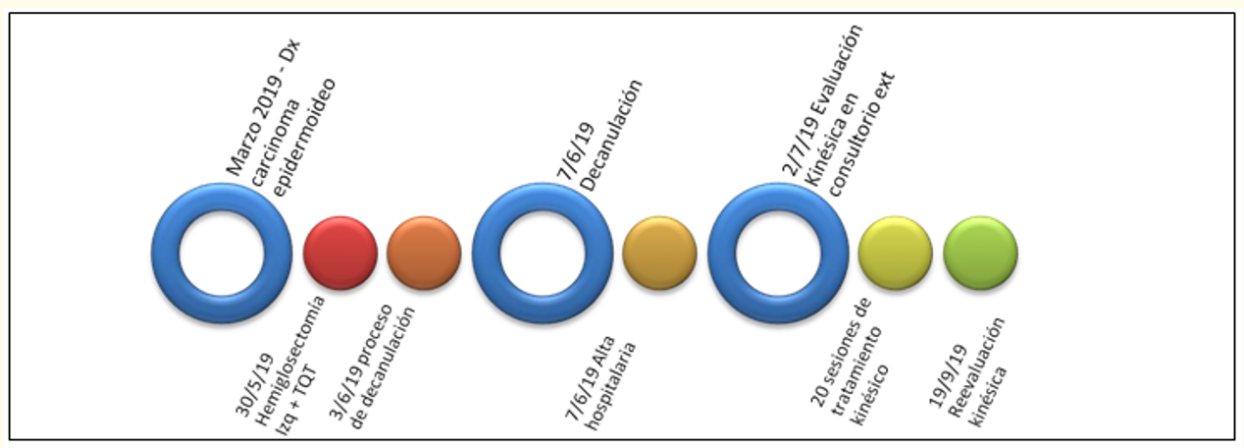

Figure 1: Timeline.

Servicio de kinesiología HOSPITAL PEDRO FIORITO
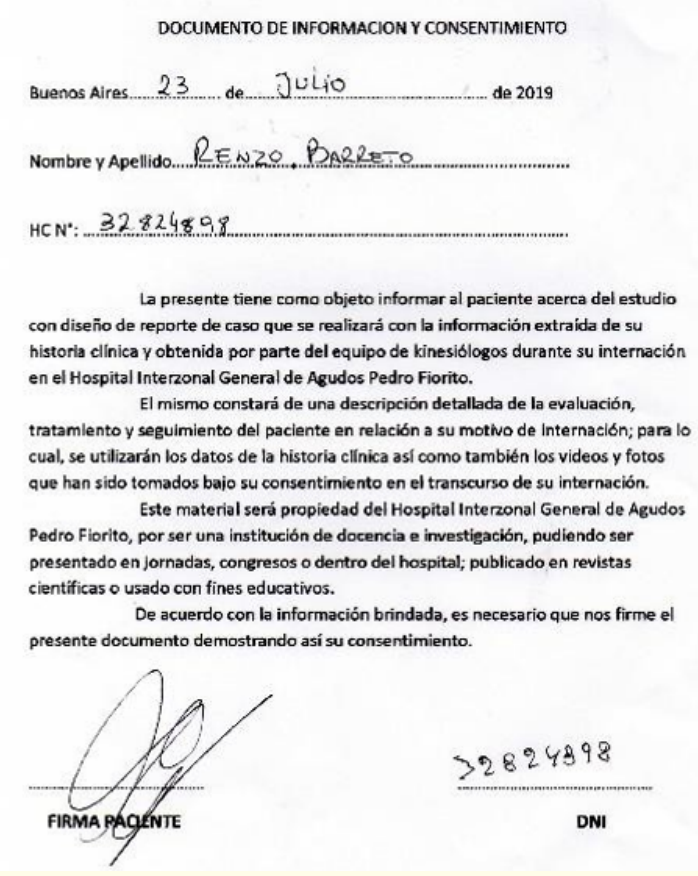

Annex 1: Informed consent. 
Initial assessment

The initial kinetic evaluation was carried out on July 2, 2019, in which the inspection shows the absence of the left anterior area of the tongue, and scar that extends from the mastoid process to the middle area of the lower jaw, passing through the floor of the mouth (Figure 2). It was hypervascularized, moderately adhered to medial predominance and limited in its movements. The measurement of the oral opening was 2.5 trof fingers birds, also finding a decrease in the protrusion of the lower jaw and in the right lateral diction. As for lingual mobility, it was limited in every way without being able to overcome the dental arch. Facial movements were preserved. Cervical right extension, tilt and rotation were markedly decreased. The patient reported feeding with liquids and semi-solids normally, difficulty with solid food and chewing only with the right arcade. He also presented moderate difficulty in the articulation of the word and in the control of saliva in the oral cavity. As for pain, it was evaluated with a numerical scale, being zero both at rest and during feeding. The complementary studies presented by the patient included a ct scan of facial massif and neck with contrast dated $11 / 7 / 2019$ that evidenced asymmetry of light at the oropharynx level, with blockage of the fatty planes to the left, without evidence of pathological enhancements after the administration of intravenous contrast. Absence of left submaxillary gland and hyperdense images were observed in this topography and alteration of the structure with increased thickness of the soft tissue in the left submaxillary region. With respect to the functional evaluation of swallowing, a video swallow was performed on 07/18/2019in which alteration of lingual movements is reported and for the assembly of the bolus in the oral stage, good velopalatine mobility, oral phase time with premature overturning, retention at the level of valecules and piriform breasts and good opening of the upper esophageal phincter, in addition there was no passage of material to the airway. Finally, the European Organisation for Research and Treatment of Cancer Quality of Life Questionnaire Head and Neck (EORTC QLQHN35) was taken, which obtained a score of 12 points out of 35 . The items reported were: pain in the jaw, inconveniences to swallow solids and to talk to other people and by telephone, difficulty eating in front of others and enjoying meals, trouble going out in public, opening mouth, dry mouth and weight loss.

\section{Kinetic treatment}

The objective was to rehabilitate the oral stage of swallowing. To reach this goal, the treatment focused on the functionality of the

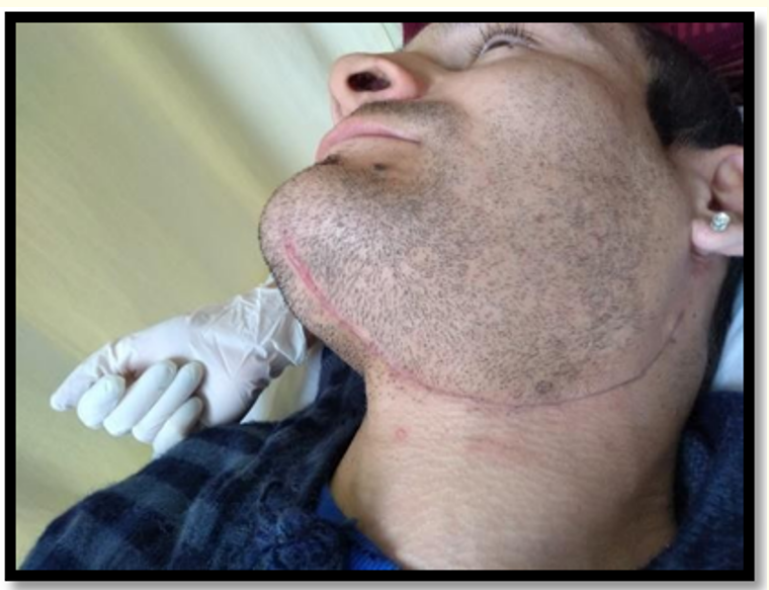

Figure 2: Scar condition at the beginning of treatment.

stomatognathic system through the improvement of chewing, lingual, cervical movements and the mobility and sensitivity of the scar.

The patient attended the kinesiology service where he performed sessions of 1 hour duration, with a frequency of twice a week. During the first, the treatment was focused on reducing the retraction of his scar, for which mobilizations were made in different directions throughout it to reduce adhesions in times of 2 minutes. In addition, negative suction was performed using a syringe for 3 minutes to mobilize the deep planes, in terms of sensitivity we worked intra-and extraorally. To increase the ranges of masticatory movements, intra and extraoral relaxation maneuvers, trigger points of masseteros muscles, pterygoids, TMW traction, pumping and muscle energy were performed ( 3 sets of 3 repetitions). The opening, diction and protrusion movements of the mouth were worked on through elongations and active exercises in front of the mirror ( 3 sets of 10 repetitions). For mobility and lingual strengthening, passive, active and anti-resistance exercises were carried out with a low tongue and the Masako maneuver ( 2 sets of 4 repetitions). As for cervical flexibility, active flexo-extension exercises, inclinations and rotations ( 2 sets of 10 repetitions) and different muscle energy maneuvers, anchors, and elongations were performed. Some of the exercises performed can be seen in figures 3 - 7. The patient received a daily exercise plan and home guidelines (Annex 2) which was supervised at each session. 

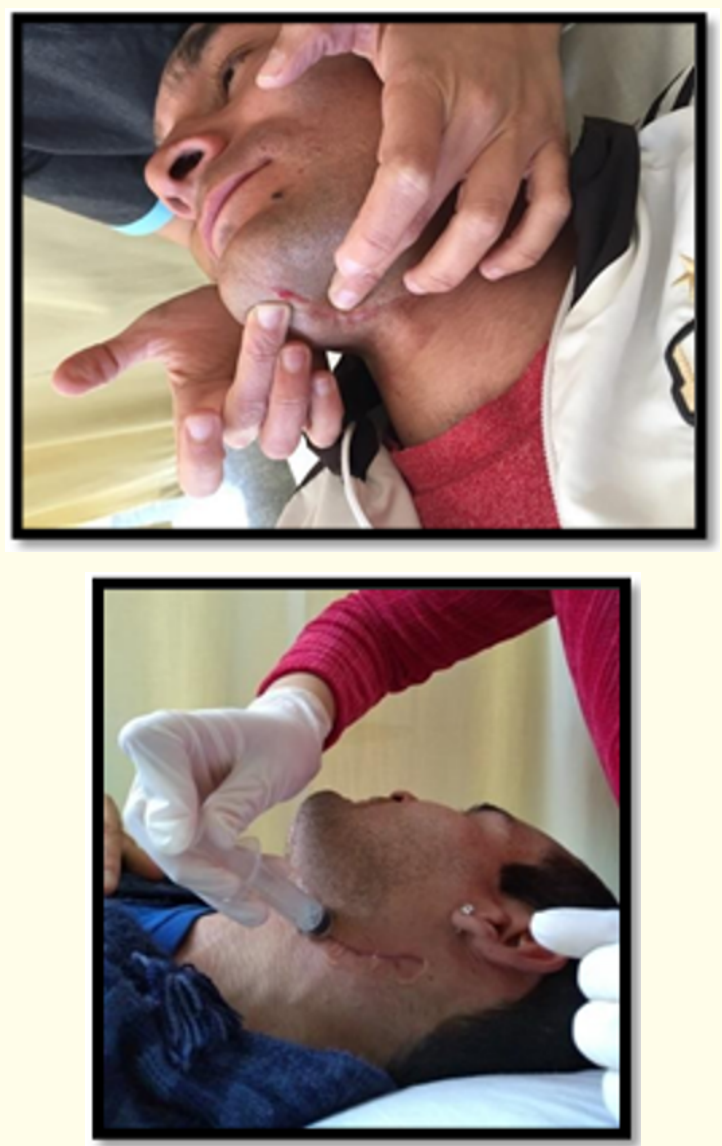

Figure 3: Mobilization and negative suction on the scar.

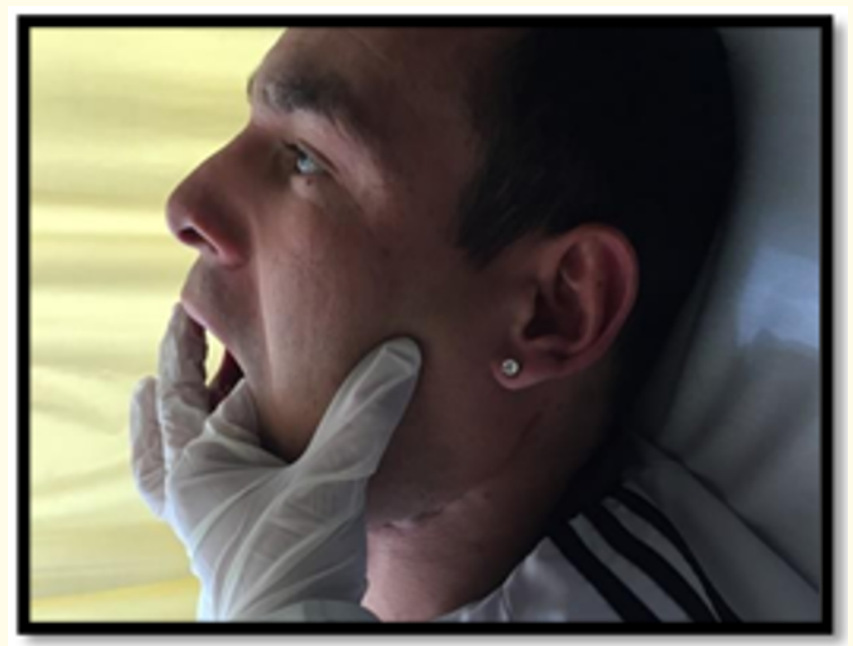

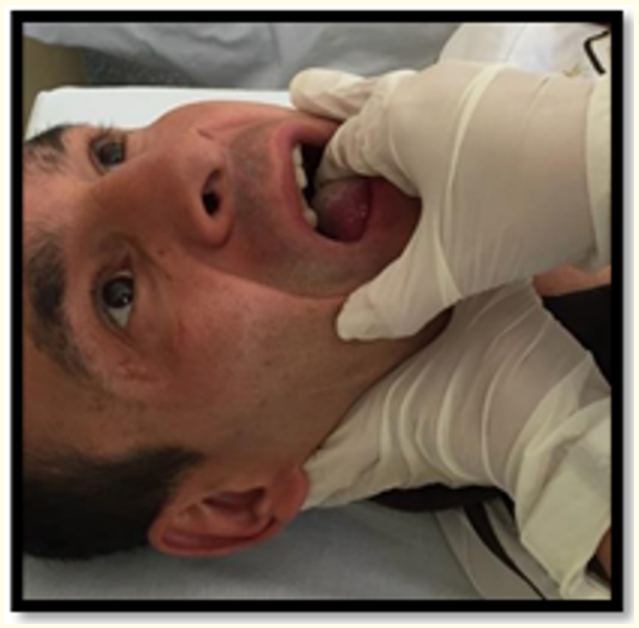

Figure 4: Streching of masseter muscle. Anti-resistance lingual movement.
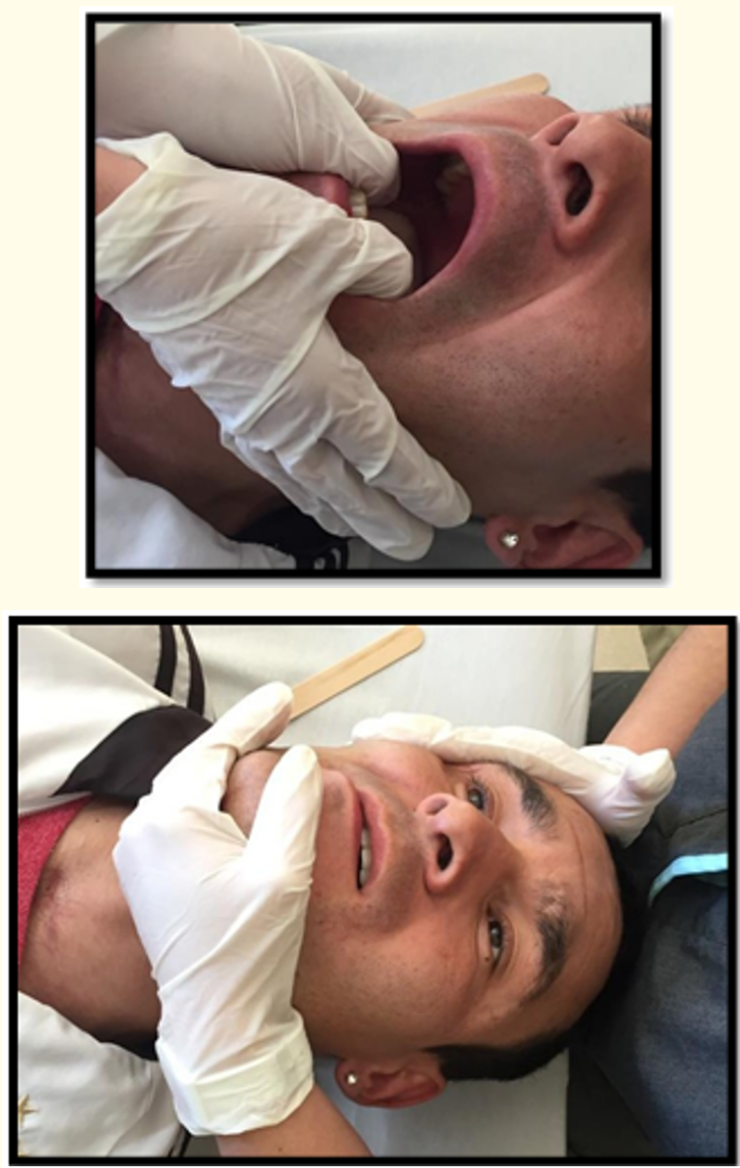

Figure 5: Muscle energy in opening and diction. 

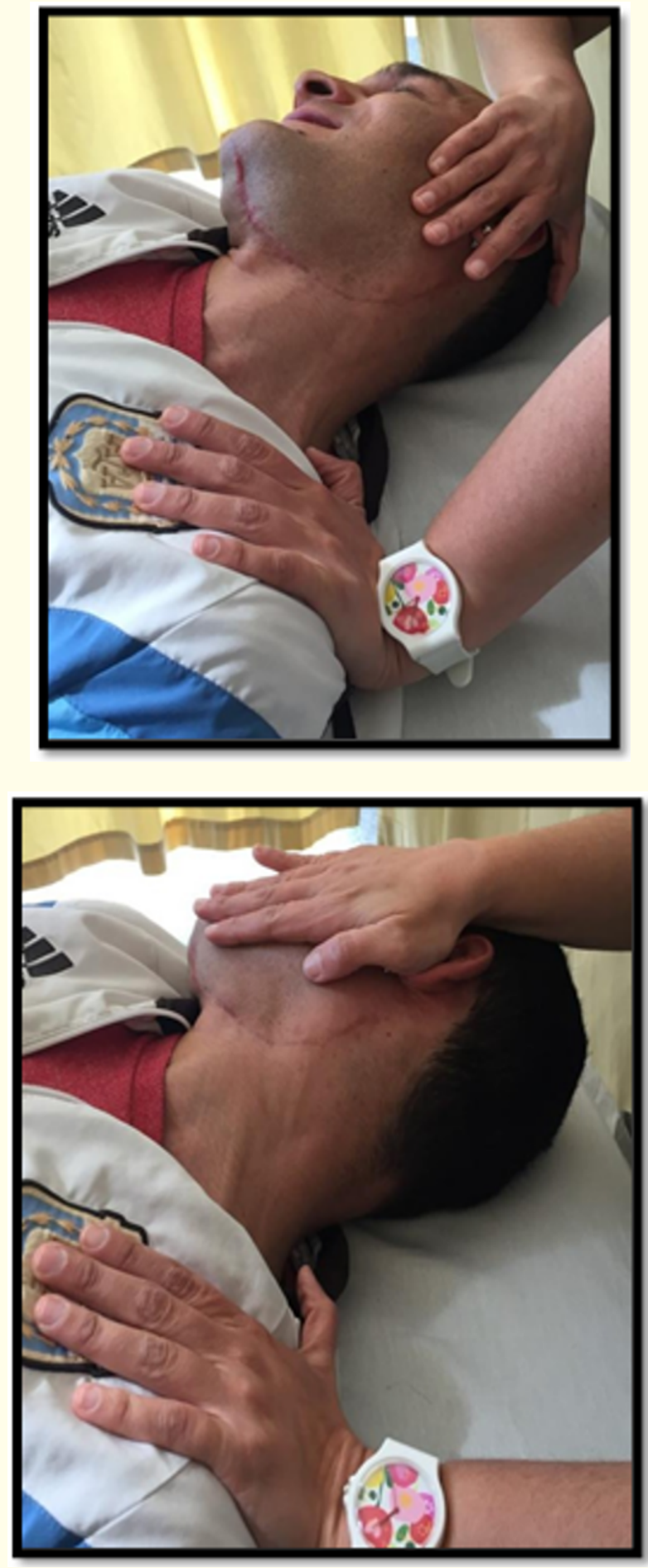

Figure 6: Elongation of tilting and rotating neck muscles.
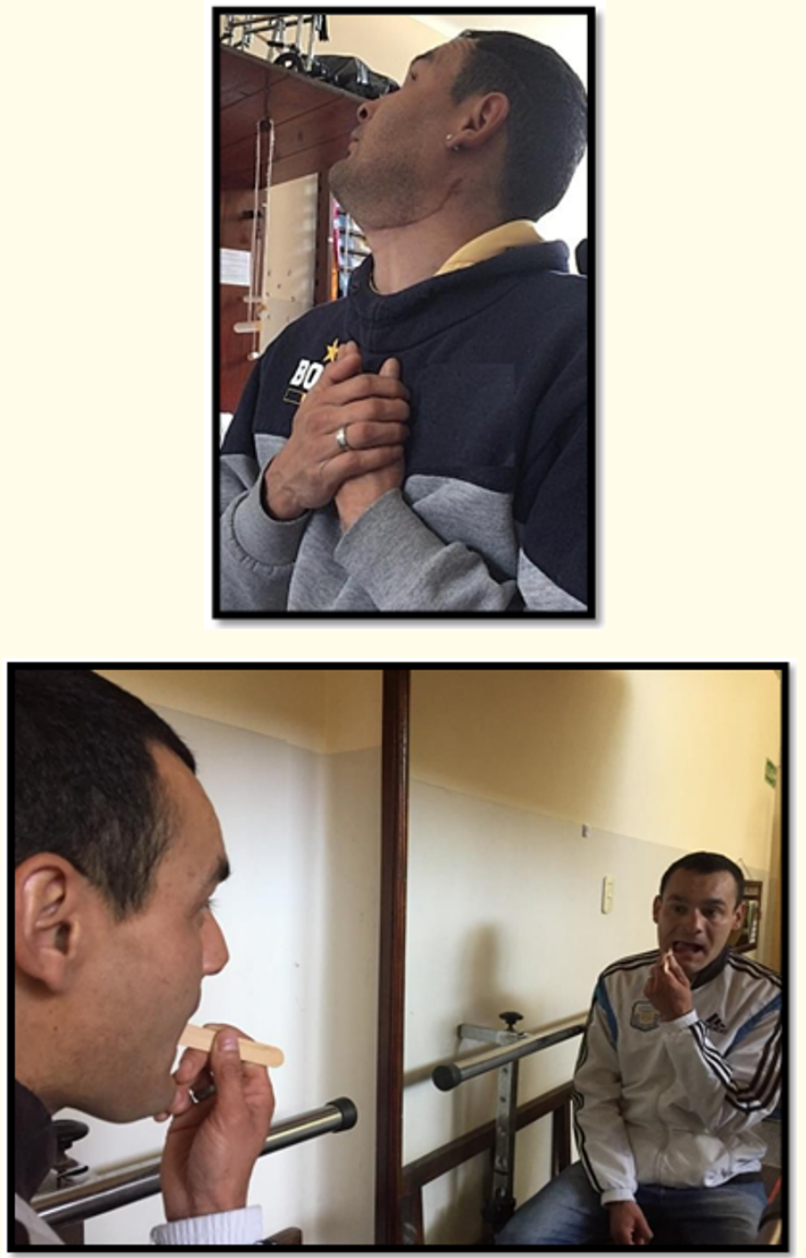

Figure 7: Auto elongation of inclination and cervical extension. Lingual mobility in front of the mirror.

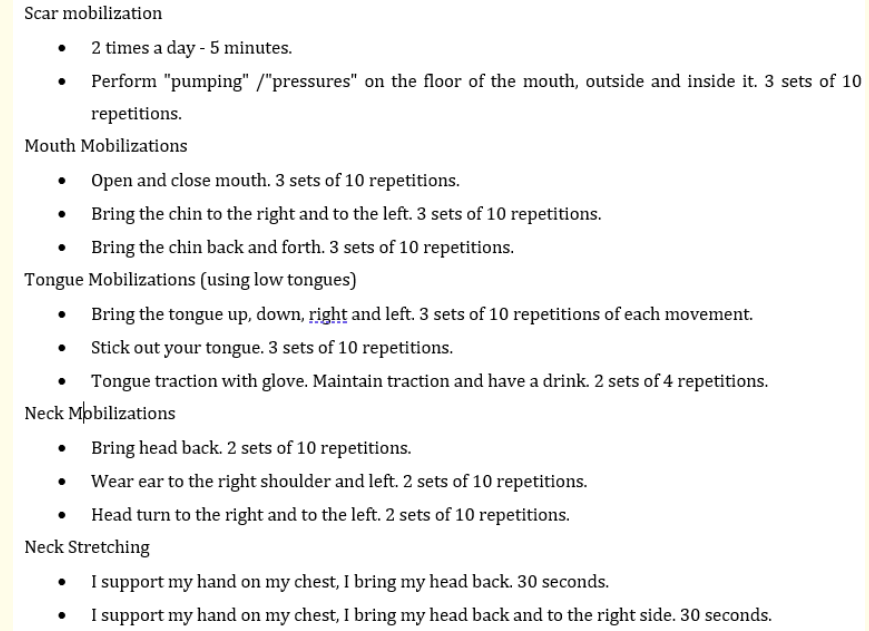

Annex 2. Home exercise plan. 


\section{Reevaluation}

It is performed on 19/09/2019 after 20 sessions of kinesiology. The scar was mobile, without adhesions or pain to the mobilization. The measurement of the oral opening was 3 fingers and the anterior protrusion exceeded the dental arch. As for lingual mobility, it was limited in the upper and right lateral direction. With regard to cervical mobility, both the left rotation and the cervical extension were slightly diminished, referring to the patient previous myofascial tension when performing the extensor movement. The other cervical movements were within normal ranges of motion. The patient reported feeding properly with all consistencies and presenting mild difficulty in the articulation of the word. He reported no alterations in zaliva control or pain. A new video swallowing with fecha was performed on 19/09/2019 in which improvement in the assembly of the bolus in the first stage and less retention in valecules and piriform breasts is reported with respect to the previous video swallowing.

Regarding quality of life, the EORTC QLQ-HN35 questionnaire had a ratio of 0 out of 35 , so the patient did not present any problems in the different items evaluated.

Figures 8 - 11 show the evolution from the initial evaluation to the re-evaluation in different measured variables.

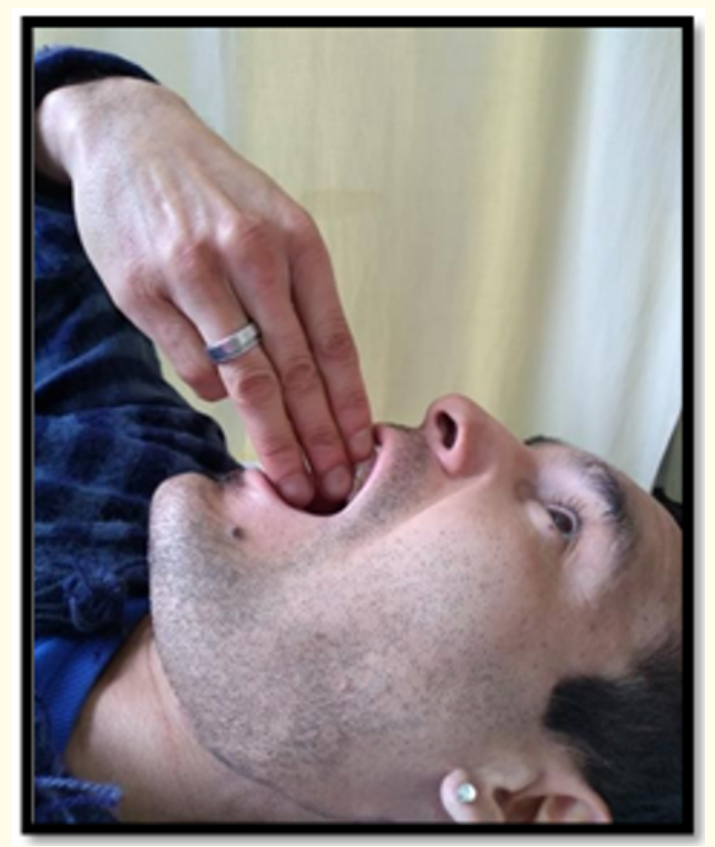

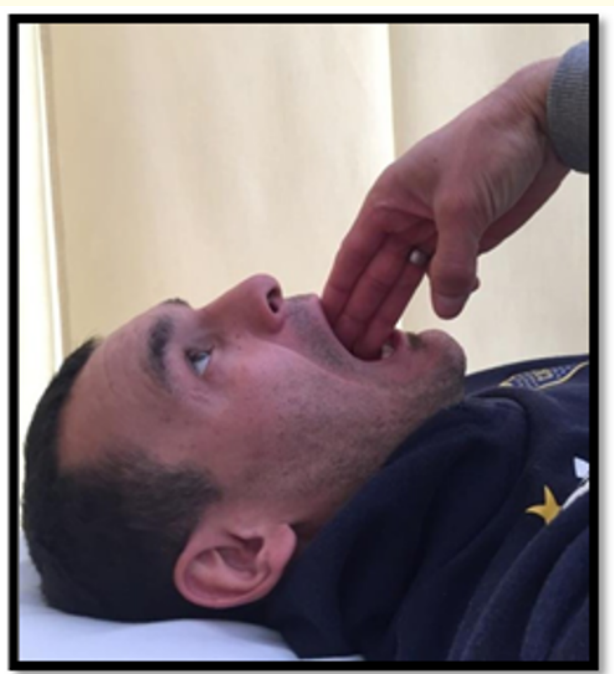

Figure 8: Oral opening at the beginning and end of treatment.
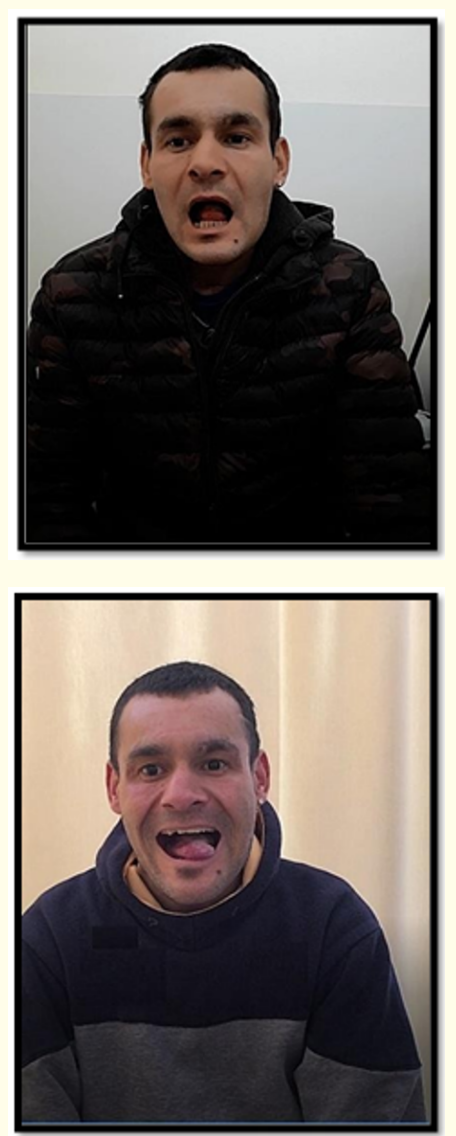

Figure 9: Lingual protrusion at the beginning and end of treatment. 

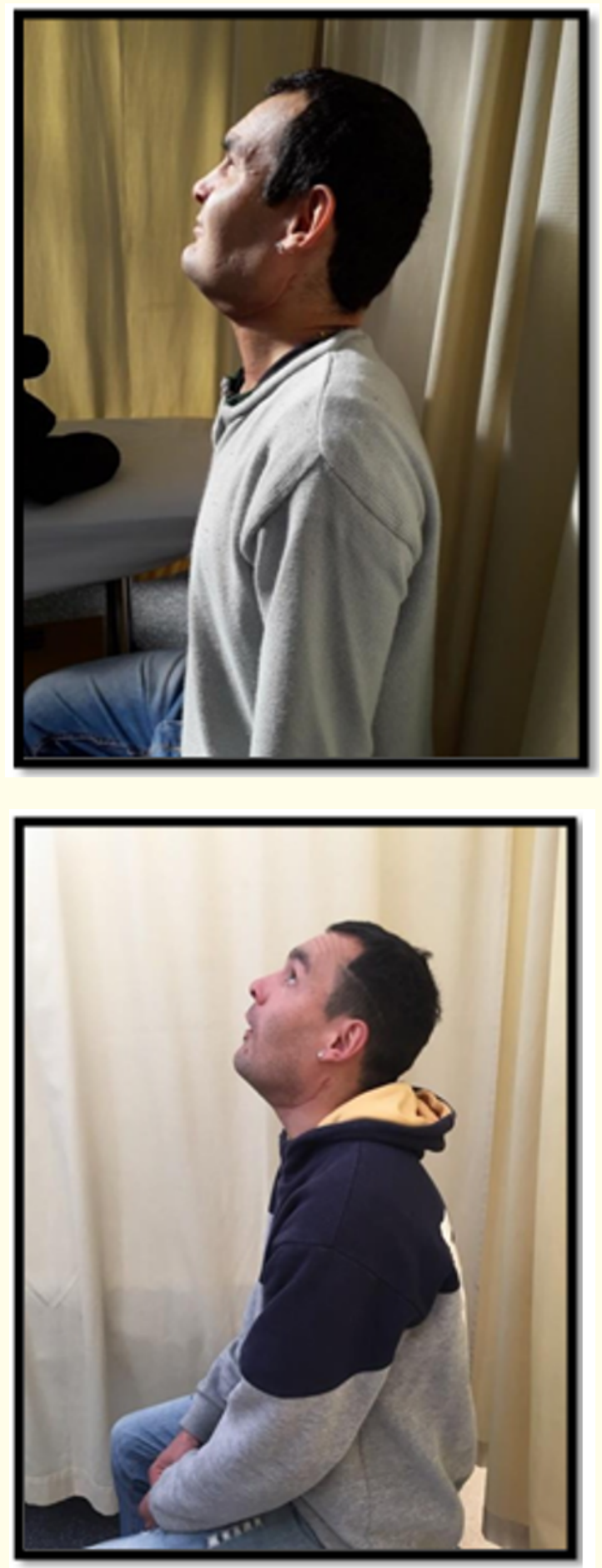

Figure 10: Cervical extension at the beginning and end of the treatment.
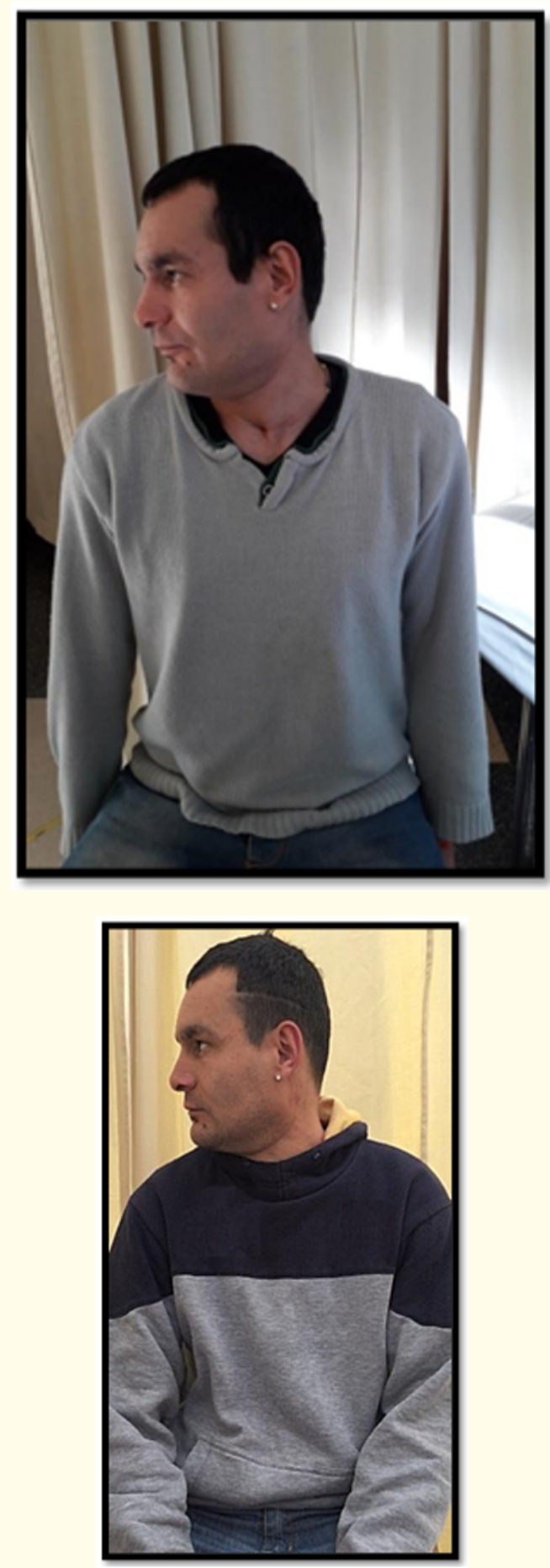

Figure 11: Right cervical rotation at baseline and completion of treatment. 


\section{Discussion}

In the present study, the case of a patient with left hemiglosectomy without indication of lingual reconstruction was described. One of the strengths of the work is that the literature collected only described treatments for patients with pre - and post-reconstruction hemiglosectomy, and no studies referring to patients such as the one reported were found. In addition, the global approach of an entire system and not only of local analytical exercises is highlighted. The overall approach to treatment achieved not only to meet the proposed objective after the initial evaluation but also to improve other functions of the stomatognathic system that were not planned in the first instance, such as the improvement expressed by the same patient of the articulación of the word, although at all times it was insisted with an inter consultation with phonoaudiology, the benefit of kinetic treatment impact on that function. It is also highlighted as a strength the good adherence of the patient and the fulfillment of the pautas indicated for the home that were always supervised by the treating kinesiologists. Among the limitations of this study are the lack of objective measurements through more reliable instruments for the variables of cervical mobility, lingual and masticatory movements. In addition, the EORTC QLQ-HN35 quality of life questionnaire is not validated in Spanish, which may have generated errors in the process of translation and interpretation of the patient of the items evaluated. In comparison with the cited literature, strength and resistance exercises were performed for the musculature and mobility of the tongue, floor of the mouth and jaw as described by Varjao ${ }^{6}$ and Lablance [7]; although other approaches were also added, which could favor the evolution of the patient. One of them was the mobilization and negative pressure with syringe of the scar to reduce adhesions of the same and with that improve its range of oral and cervical mobility. In addition, masticatory muscles were addressed with techniques not described in the cited literature such as intra-and extraoral relaxation, trigger points, tractions and muscle energy. We found no studies that describe the approach to cervical flexibility after a hemiglosectomy, however, active exercises, myofascial anchoring, muscle energy and cervical elongations were performed since the neck and shoulder girdle are part of the stomatognathic system as described by Rocavado [9]. The presentation of this work shows that the impact of hemiglosectomy includes not only lingual mobility in isolation but also restrictions on cervical mobility, scarring, masticatory movements and swallowing. Because of this, a comprehensive and functional kinetic approach to the system responsible for these functions is necessary for the correct evolution of this type of patients.

\section{Conclusion}

The present study demonstrated a favorable evolution in the functionality of the stomatognathic system in a patient with hemiglosectomy without indication of dental prosthesis or lingual re- construction, after having carried out a rehabilitation plan in the external office of higa Pedro Fiorito de Avellaneda.

\section{Bibliography}

1. Garcia-Peris P., et al. "Long-term prevalence of oropharyngeal dysphagia in head and neck cancer patients: impact on quality of life". Clinical Nutrition 26.6 (2007): 710-717.

2. Petersen P E. "Oral cancer prevention and control-the approach of the World Health Organization". Oral Oncology 45.45 (2009): 454-460.

3. Nieto Ramírez L E and Pedraza Borrero C I. "Microsurgical reconstruction of tongue". Chilean Journal of Surgery 68.2 (2016): 180-185.

4. Bachher G K., et al. "Effective rehabilitation after partial glossectomy". Indian Journal of Otolaryngology and Head and Neck Surgery 54.1 (2002): 39-43.

5. Mannelli G., et al. "Classification of tongue cancer resection and treatment algorithm". Journal of Surgical Oncology 117.5 (2018): 1092-1099.

6. Varjão F M. "Myofunctional therapy as an aid to prosthodontic treatment after hemiglossectomy: A clinical report". The Journal of Prosthetic Dentistry 107.5 (2012): 284-287.

7. LaBlance G R., et al. "Rehabilitation of swallowing and communication following glossectomy". Rehabilitation Nursing 16.5 (1991): 266-270.

8. Zhen Y., et al. "Efficacy survey of swallowing function and quality of life in response to therapeutic intervention following rehabilitation treatment in dysphagic tongue cancer patients". European Journal of Oncology Nursing 16.1 (2012): 54-58.

9. Chiavaro Norma. "Stomatognathic functions and dysfunctions". Akadia Editorial Bookstore (2011): 4-5.

\section{Assets from publication with us}

- Prompt Acknowledgement after receiving the article

- Thorough Double blinded peer review

- Rapid Publication

- Issue of Publication Certificate

- High visibility of your Published work

Website: www.actascientific.com/ Submit Article: www.actascientific.com/submission.php Email us: editor@actascientific.com Contact us: +919182824667 